\title{
OVERVIEW OF WIND LIDAR TECHNIQUES AND CURRENT RELATED DEVELOPMENTS AT THE TECHNICAL UNIVERSITY OF CATALONIA
}

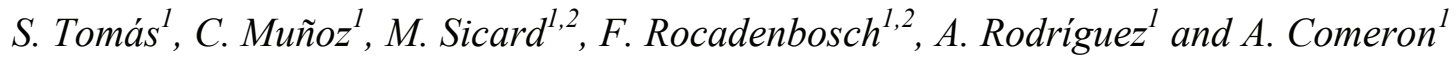 \\ ${ }^{1}$ Remote Sensing Lab. (RSLAB), Dep. of Signal Theory and Communications, \\ Universitat Politècnica de Catalunya \\ C/Sor Eulalia de Anzizu sn., D4-011 \\ 08034 Barcelona, Spain. \\ ${ }^{2}$ Institut d'Estudis Espacials de Catalunya - Centre de Recerca de l'Aeronàutica i de l'Espai / Universitat \\ Politècnica de Catalunya
}

\begin{abstract}
The Lidar group in the Technical University of Catalonia is actively working in wind lidar techniques. An extensive review of such techniques is presented. A special emphasis is made on the direct Doppler technique and the direct motion technique with a lines-of-sight scanning pattern. Current developments are also mentioned.
\end{abstract}

Index Terms - Doppler lidar, correlation lidar, wind detection, lidar design, lidar signal processing

\section{INTRODUCTION}

Spatially resolved wind speed measurements from a remote location are required in manifold areas: aviation safety, studies for wind farms installations, climate research or weather forecasting. They offer a spatial and temporal resolution higher than the traditional in situ methods. The most significant remote sensing systems to obtain rangeresolved wind measurements are sodar, radar and lidar.

The complementarity of these techniques is well established. The lidar technology is able to work in clear air conditions and offers much better spatial resolution. On the other hand, rain, clouds or even fog impair the lidar sensing, where radars are able to measure. Sodar techniques are low range limited but they are cheaper and the involved technology is well developed and robust.

Regarding the basic measurement used to obtain the wind speed, wind lidar techniques can be classified in Doppler detection and direct motion techniques. The first ones measure the Doppler shift in the lidar return while the second ones look for a disparity between power signals in range and/or time dimensions. The Lidar Group of the Remote Sensing Lab of the Technical University of Catalonia is currently working in both areas of wind lidars.

\section{DOPPLER DETECTION}

The operation of a Doppler lidar depends on the detection of the Doppler shift in the frequency of the backscattered radiation from either atmospheric aerosol particles or molecules, which is directly related to the wind speed in the line-of-sight (LOS) direction (radial wind).

\subsection{Coherent detection lidar}

In these systems the Doppler shift is measured using radiofrequency heterodyne techniques. Their operation is based on mixing the return signal with a frequency-stable local oscillator (a laser beam) on a wideband, square-law optical detector and on signal processing techniques used to estimate the spectral peak of the beat signal.

The main advantage of coherent lidars is their high sensitivity in the optical detection. Applying enough local oscillator power the quantum limit situation can be achieved. In these conditions the shot noise of the local oscillator beam dominates any other noise of the system and the signal to noise ratio is the maximum possible [1].

Their main drawback is their limitation to the infrared region, where Rayleigh scattering is weak. Consequently their use in zones without Mie scattering becomes difficult and, as a result, it can be said that, although they are the preferred when measuring winds in continental, low altitude regions where the aerosol content is high, they will not be suited to measure winds at high altitudes in the middle atmosphere or, in general in any region with low aerosol loading conditions [2].

\subsection{Direct Detection Doppler lidar}

Direct detection based Doppler wind lidars use an optical frequency discriminator or a spectrum analyzer to convert the Doppler frequency shift to a change in optical intensity, or to an intensity/power spatial distribution, which is 
directly detected. The return optical signal is spectrally resolved prior to detection. The optical frequency discriminators used include mainly 3 types: edge filters, fringe pattern imaging and atomic absorption lines of certain atmospheric constituents.

The systems based on edge filters use the quasi-linear zone of the slope of high resolution optical filters to convert frequency shifts in intensity changes. There are two variants of this technique. In the edge technique, a single optical filter is tuned so that the emitted frequency is located on the most sensitive zone of the filter frequency response. In the double edge technique, two optical filters are tuned to locate their opposite slopes symmetrically at the emitted frequency, improving in such way the sensitivity and the accuracy of the measurement.

The fringe imaging instruments resolve the backscattered Doppler shift by projecting the interference pattern from an optical interferometer, usually a Fabry-Perot interferometer, onto a multichannel detector. The Doppler shift is obtained observing the angular displacement of the fringes location.

The resonance fluorescence Doppler lidars use the atomic absorption lines of different atmospheric constituents, like $\mathrm{Na}, \mathrm{K}$ and $\mathrm{Fe}$, as natural frequency analyzers using the resonance fluorescence from atoms. These systems measure wind velocity in the mesosphere and lower thermosphere from the Doppler shift of the atomic absorption spectrum of the chosen constituent [3].

The main advantage of the direct detection Doppler wind lidars is that, since they do not need a frequency stable $\mathrm{CW}$ local oscillator laser for the detection, they can operate in the UV region by using non linear optical devices to double and triple the fundamental frequency of the pulsed laser. This permits to operate in regions where there are not aerosols by detecting the Doppler shift in the molecular return. Therefore, they are the best suited for measurements at high altitudes and at global scale [2].

\subsection{Present work at the UPC: wind Doppler lidar}

The lidar group of the Remote Sensing Lab. of the Technical University of Catalonia is currently working in the development of a wind Doppler lidar using the edge technique.

The edge technique principle consists in comparing, for every resolution cell, the transmittance of a high resolution optical filter at two different frequencies: that of the emitted light and that of the received one. From these values and using a previous calibration of the filter frequency response, the Doppler shift and, consequently, the speed of the target that has provoked it can be derived. To do this in an effective way it is indispensable that the filter is tuned so that the wavelength of the transmitting laser is located on the most sensitive zone of the slope of the filter, where small frequency changes, caused by Doppler shift, produce big changes in its transmission (Fig. 1) [4].

In order to assess this measuring technique and to test the performance of all the subsystems in controlled conditions, avoiding those difficulties related with the use of pulsed light and with the detection of atmospheric signals, a continuous-wave solid-target prototype has been designed and built. Figure 2 shows the scheme of the prototype.

The transmitting source is a continuous-wave laser. After having been conditioned, a beam splitter (BS1) splits the output beam in two: one towards the target and the other is used as the reference of the emitted frequency (reference channel). A polarizing beam splitter (BS2), a quarter wave plate and a focusing system allow illuminating a spot on the paper surface of a rotating wheel, collecting part of the frequency shifted backscattered radiation and leading it to the measuring subsystem (Doppler channel).

An optical chopper commutates alternatively reference and Doppler channels. Both channels are coupled into graded index multimode optical fibres. A low cost $2 \times 2$ optical fibre coupler divides the optical power from each channel in two outputs. One of them goes to an APD based optical receiver and the other one to the high resolution optical filter, in this case a tuneable Fabry-Perot interferometer, and to a second receiver. A mode scrambler is used to assure the same filter response for both channels. A collimating lens and a diaphragm condition the incident light to the interferometer. A 16 bit, up to $250 \mathrm{kS} / \mathrm{s}$, multifunction acquisition card acquires both receivers output voltage and a personal computer processes the data.

As a consequence of the unavoidable drifts in both, the laser wavelength and the resonance frequencies of the interferometer, a real-time control loop has to be set in order to compensate them and keep the proper relative position between the emitted frequency and the filter slope. This subsystem acts on the interferometer, which is equipped with piezoelectric cells to fine tune its cavity length.

\section{DIRECT MOTION DETECTION}

The direct motion detection is a technique for elastics lidars provided with a scanning capability. It is a technique that retrieves the wind velocity fields from the displacements of aerosol concentration patterns in pairs of images. The technique reliability depends on the spatial and temporal variability of the aerosol content, which is chiefly restricted to the atmospheric boundary layer. The aerosol concentration, which is almost proportional to the received power, has a fluctuating spatial distribution that responds fast enough to the wind changes. From those fluctuations, there are contrasted aerosol inhomogeneities that are mainly advected, although diffused as well by the wind flow [5].

The motion is detected by means of a comparison between signals taken at different positions and/or at 
different times. This gives two different approaches, depending on the scanning patterns available.

\subsection{Lines-of-sight pattern sensing}

The first approach consists in pointing a laser beam in several directions and scan back-and-forth. The choice of the elevation angle, either the zenith or a slant line of sight, leads to two different techniques. The first case is an adaptation of the spaced antennas technique: three to five directions are used to perform the full correlation analysis. In the second case, a near-to-ground direction is considered and the azimuth scans are realized over a narrow separation varying between from 1 to 5 degrees [6].

The comparison between the signals, the temporal evolution of the range-square corrected signals as a function of range, is done by a time or a spatio-temporal crosscorrelation, or a related coherence function. The lag of the cross-correlation maximum gives an apparent wind motion, which also contains the diffusive effect of the turbulence. However, the intersection with an auto-correlation gives an estimate of the true advection [7].

The scanning can be avoided if multiple beams are used. An innovative approach uses an array of beams [8]. The signals are analyzed in the frequency domain to determine the lag made by the wind. This technique constitutes an alternative to the template-matching approaches stated above.

\subsection{Area/Volume sensing}

The area/volume methods are based in motion detection in satellite imaging. When large zones $\left(\mathrm{km}^{2}\right)$ are required and faster systems are available, scanning patterns can help to enlarge the detected area or produce a volume image by stacking the areas detected [9]. The area images are obtained for different times (up to 30s). A bi-dimensional spatial cross-correlation determines the disparity between horizontal images. The spatial lag is found by interpolation around the maximum.

\subsection{Present work at the UPC: correlation wind lidar}

The motion detection technique developed at the UPC is based on the study by Buttler et al. [6]. It employs a 2D space-time correlation between pairs of slanted LOS. Since the technique is well established, the main efforts are based in two topics: assessment of the optimal measurement procedure and improvement of the wind detection algorithms.

The existence of several measurement trade-offs in the LOS sensing methods makes them quite sensitive to the angular separation. One remarkable parameter is the number of integrated pulses in each LOS: the lesser, the faster the scan is and the higher time resolution is achieved; on the contrary, the higher, the better the SNR is, and the maximum range, and height, increases. One main drawback in all these techniques is that pulse averaging, which limits the time resolution.

In the technique developed at UPC, before the transverse wind across the LOS is determined, a processing step to remove noise and enhance the significant features is made with a median filtering to preserve the boundaries without smoothing. Then, a match procedure is performed. An image feature analysis permits to decide the meaningful regions before considering a variable window size to perform the match. The pair of images for the respective LOSs is chosen so as to maximize the correlation.

Then, the correlation function is evaluated for a certain threshold, and the centroid of the resulting almost-elliptical level curve is determined. By geometry, the separation between LOSs is determined and is divided by the lag of the time coordinate of the centroid respect to the origin. This ratio is the apparent transverse wind velocity.

An analog procedure but applied to the auto-correlation function gives true elliptical level curves whose orientation in the range-time axis is the indicator of the radial wind [7].

An example of a horizontal wind field obtained in Barcelona on July $13^{\text {th }} 2008$ at 1915 UTC is shown in Fig. 3 . The azimuth orientation was SW $\left(225^{\circ}\right)$, the azimuth angular separation between both LOSs was $3^{\circ}$ and the elevation was $20^{\circ}$. The height resolution is $5 \mathrm{~m}$.

\section{SUMMARY}

All the atmospheric wind lidar techniques have been presented. Nowadays the direct detection Doppler is the best suited technique to high altitudes and space-born systems; the coherent Doppler is the most sensitive but technologically restricted to certain scenarios. In parallel, the alternative that correlation methods offer can make the elastic lidars able to provide wind measurements with some investment in scanning systems, which is in fact also required for the Doppler systems, although mostly their performance rely on signal interpretation algorithms as well as their further developments.

\section{ACKNOWLEDGEMENTS}

The work presented here was supported by the European Union and FEDER funds under the EARLINET-ASOS project (EU Coordination Action, contract no025991 (RICA)); by the European Space Agency under the project 21487/08/NL/HE and by the MEC (Spanish Ministry of Education and Science) and FEDER funds under the project TEC2006-07850/TCM, and the Complementary Actions CGL20007-28871-E/CLI and CGL2006-26149-E/CLI.

S. Tomás and M. Sicard are grateful to the MEC for the FPI pre-doctoral fellowship and for the Ramón y Cajal position that they respectively hold. 


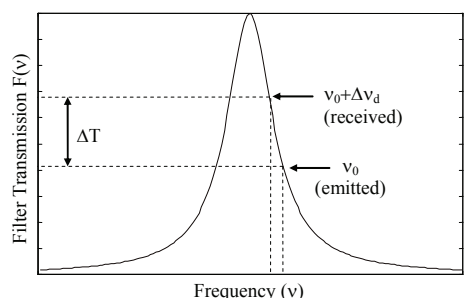

Fig. 1. (Above) Principle of the edge technique for a high discriminating filter.

Fig 2. (Right) Scheme of the prototype currently designed at the UPC.
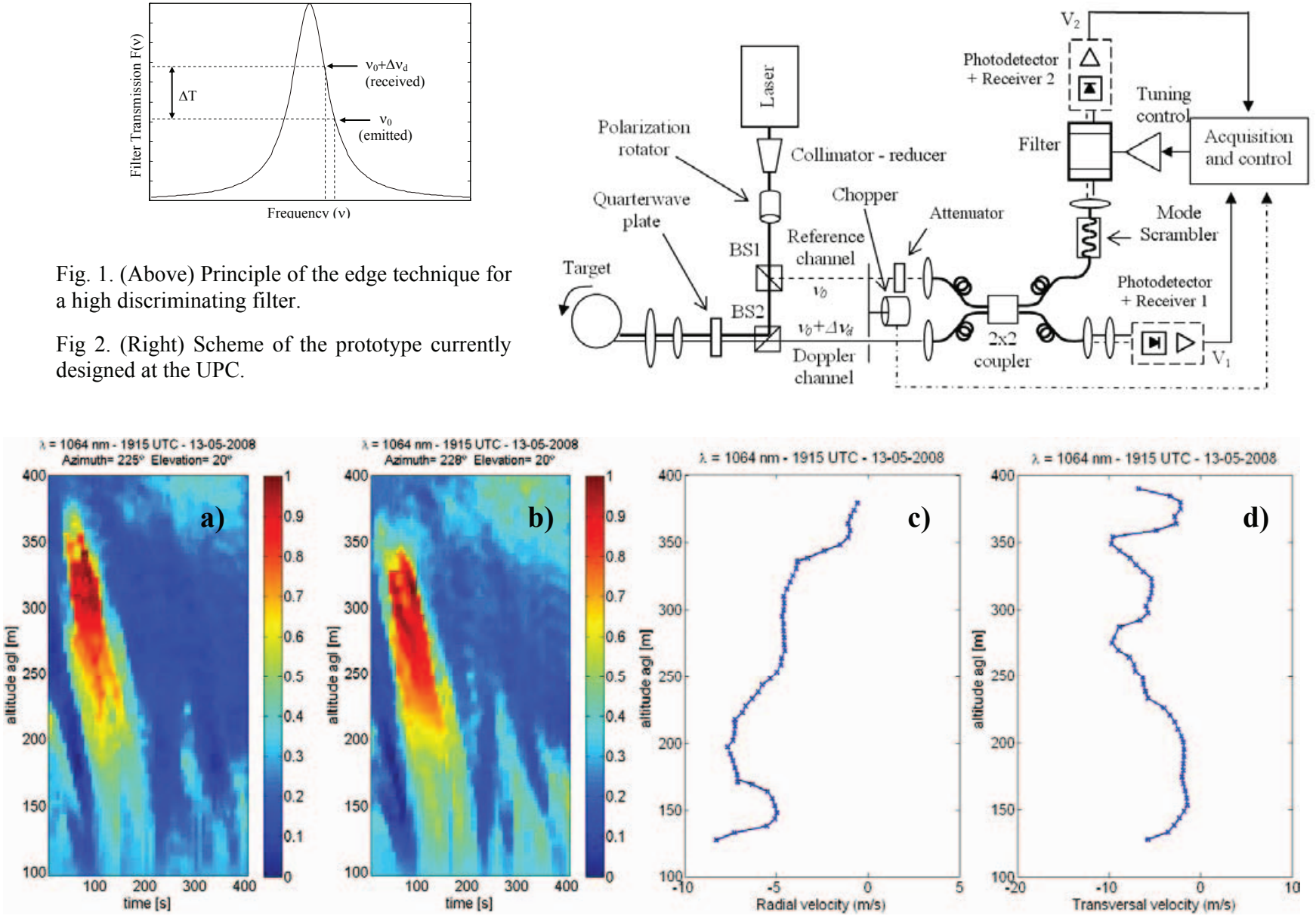

Fig. 3. a) and b) Height-time images of aerosol patterns after subtracting the temporal averaged lidar profile for each one. c) Radial wind velocity with negative values meaning towards the lidar and d), tranversal velocity with positive values from the $225^{\circ}$ to $228^{\circ}$ pointing.

\section{REFERENCES}

[1] A. Rodriguez. "Sistemas Lidar coherentes e incoherentes de baja potencia para la detección de velocidad de blancos sólidos". Doctor-Engineer Thesis, Technical University of Catalonia, Barcelona, October 1998.

[2] P. Hays, M. Dehring, L. Fisk, P. Tchoryk, I. Dors, J. Ryan, J. Wang, M. Hardesty, B. Gentry, F. Hovis, "Space-based Doppler winds lidar: a vital national need". In response to National Research Council (NRC) Decadal Study Request For Information (RFI). May 2005.

[3] X. Chu, W. Huang, and J. Wiig, "High-Resolution DopplerFree Polarization Spectroscopy for Novel Doppler Lidars for Environmental and Atmospheric Sciences". CIRE's Annual, Institute-wide Symposium, Boulder, Colorado (USA), April 2007.

[4] C. L. Korb, B. M. Gentry, and C. Y. Weng, "Edge technique: theory and application to the lidar measurement of atmospheric wind", Applied Optics, 31(21), pp. 4202-4213 (1992).
[5] V.E. Zuev, Yu. M. Vorevodin, G.G. Matvienko and I.V. Samokhalov, "Investigation of structure and dynamics of particulate inhomogeneities in the ground layer of the atmosphere," Applied Optics, 16(8), pp. 2231-2235 (1977).

[6] W. T. Buttler, C. Soriano, J M. Baldasano and G. H. Nickel. "Remote sensing of three-dimensional winds with elastic lidar: explanation of maximum cross-correlation method," BoundaryLayer Meteorology, 97, pp. 305-328 (2001).

[7] G. J. Kunz, "Wind Measurements with an Incoherent Lidar," TNO Physics and Electronics Laboratory, FEL-93-A040, August 1993.

[8] W.E. Eichinger and J.R. Krieger, "Multiple beam lidar system for wind measurement," United States Patent, No US 6,646,725 B1, November 11, 2003.

[9] J.L. Schols and E.W. Eloranta, "Calculation of area-averaged vertical profiles of the horizontal wind velocity from volumeimaging lidar data," J. Geophys. Res, 97, pp.18,395-18,407 (1992). 